DOI: 10.12957/demetra.2018.32455

\title{
Alimentos ultraprocessados congelados e ready to heat versus tendência de saúde e bem-estar em Porto Alegre, Rio Grande do Sul
}

\section{Ultra-processed frozen and ready to heat foods versus health and wellness trend in Porto Alegre, Rio Grande do Sul state}

Jéssica Petersen Kruel'

Poliana Deyse Gurak

Monica Concha-Amin²

1 Universidade Federal de Ciências da Saúde de Porto Alegre, Departamento de Nutrição. Porto Alegre, RS, Brasil.

${ }^{2}$ Universidade Federal de Ciências da Saúde de Porto Alegre, Departamento de Ciências Exatas e Sociais Aplicadas. Porto Alegre, RS, Brasil.

Correspondência / Correspondence Monica Concha-Amin

E-mail:monicac@ufcspa.edu.br

\section{Resumo}

Objetivos: Verificar se produtos alimentícios classificados como ultraprocessados congelados e ready to heat, comercializados na cidade de Porto Alegre-RS, estão sendo fabricados com atributos da tendência de saúde e bem-estar. Metodologia: O grupo de produtos analisados inclui: pizzas, lasanhas e pratos prontos como massa à bolonhesa, massa quatro queijos, risotos, nuggets, entre outros. A escolha da unidade visitada se baseou na localização predominante da classe econômica C na cidade de Porto Alegre, registrada na Análise Socioeconômica Porto Alegre 2017. Resultados: Um total de $77 \%$ da amostra geral foi classificado com excesso de gordura; $95 \%$ com excesso de sódio; $70 \%$ sem corantes adicionados intencionalmente e $78 \%$ sem conservantes. Apenas $11 \%$ foram considerados light em sódio; $74 \%$ como não fonte de fibras; e $74 \%$ não utilizam publicidade no rótulo para diferenciar seu produto no mercado. O grupo A (das marcas mais lembradas) possuiu maior peso da porção $(\mathrm{g})$, maior porcentagem vindo de gorduras totais (\%), gorduras totais (g) e fibras na porção $(\mathrm{g})$, maior relação sódio/kcal, maior número de ingredientes, de produtos light, de produtos fontes de fibras e uso de publicidade no rótulo. Já o grupo B (das marcas menos lembradas) apresentou unicamente maior quantidade de gordura trans na porção. Discussão: Diante de concorrentes com produtos menos "saudáveis" nas prateleiras do varejo supermercadista, as grandes marcas continuam apostando mais na produção e comercialização de grandes volumes do que na tendência de saúde e bem-estar. Conclusões: Os rótulos investigados não mostraram evidências para se encaixarem na linha de produtos com atributos da tendência de saúde e bemestar. No entanto, para o caso de rótulos de produtos congelados 
ready to heat das marcas mais lembradas, foi possível identificar, embora discretamente, alguns aspectos dessa tendência, tais como presença de versões light, ter mais gramas de fibras na porção e fontes de fibras, menos gordura trans na porção e não adição de aditivos intencionalmente. Dessa forma, os consumidores da classe econômica C, em Porto Alegre, têm nessa linha de produtos poucas alternativas para uma alimentação mais saudável, sugerindo maior atenção para os formuladores de políticas e práticas para melhor qualidade de vida relacionada à nutrição.

Palavras-chave: Publicidade Direta ao Consumidor. Alimentos Industrializados. Alimentos Congelados. Comportamento do Consumidor. Saúde Pública.

\section{Abstract}

Objectives:To verify whether food products classified as ultraprocessed frozen and ready to heat, marketed in the city of Porto Alegre, Rio Grande do Sul state, are being manufactured with attributes of the health and wellness trend. Methods: A descriptive observational studyof analysis of labelswas carried out at four supermarkets and at onewholeretail store from the city of Porto Alegre-RS. The group of analyzed products includes: pizzas, lasagne, ready-made dishes like Bolognese pasta, risottos, nuggets, among others. The choice of the visited unit was based on the predominant location of thelower-middle classin the city of Porto Alegre registered in thePorto AlegreSocioeconomic Analysis 2017. Results: $77 \%$ of the general sample was classified withexcess fat; $95 \%$ with excess sodium; $70 \%$ without intentionally added dyes and $78 \%$ without preservatives; $11 \%$ was considered light in sodium; $74 \%$ as non-fiber source and $74 \%$ do not use advertising on the label to differentiate the product in the market. Group A had higher weight of the portion (g), higher percentage from total fat (\%),total fat (g) and fibers in the portion (g), higher sodium/ kcal ratio, higher number of ingredients, oflight products, of products that arefiber sources and the use of advertising on the label. Group B only presented a higheramount of trans fat in the portion. Discussion: By facing competitors with less "healthy" products on supermarket shelves, large brands continue to bet more on the production and marketing of large volumes than on the health and wellness trend. Conclusions:The investigated labels did not exhibit evidences to fit into the line of products that have the attributes of the health and wellness trend. However, in group A, the most recognized brands, it was possible to discreetly 
identify some aspects of this trend, such as the presence of light versions, having more grams of fibers in the portion and sources of fibers, less trans fat in the portion and no intentional addition of additives. Thus, consumers from thelower-middle class, in Porto Alegre, have in this line of products few alternatives to a healthier diet, which suggests a greater attention to policy makers and practices for a better quality of life related to nutrition.

Keywords: Direct-to-Consumer Advertising. Industrializad Foods. Frozen Foods. Consumer Behavior. Public Health.

\section{Introdução}

As tendências de mercado estão relacionadas à conjuntura social e econômica de uma sociedade, sendo uma influência que possui durabilidade, capaz de projetar o futuro, e cujo impacto é perceptível pela mudança de comportamento dos indivíduos. ${ }^{1} \mathrm{O}$ Brasil apresenta cinco tendências na área de alimentos, as quais impactam as escolhas alimentares do brasileiro. São elas: 1) Sensorialidade e Prazer; 2) Conveniência e Praticidade; 3) Confiabilidade e Qualidade; 4) Sustentabilidade e Ética; 5) Saúde e Bem-Estar. ${ }^{2}$

Conveniência e Praticidade são decisivas na seleção de produtos alimentícios, sem discriminação entre as classes econômicas. ${ }^{3,2}$ Isso se justifica, pois atualmente a mulher está inserida no mercado de trabalho, a população tem vida atarefada, trabalha em tempo integral e dispõe de pouco tempo para a alimentação da família., ${ }^{24}$ Assim, os alimentos industrializados prontos para consumo se tornam escolhas-chave, por agregarem praticidade às refeições. ${ }^{2,4-8}$

No Brasil, a tendência de saúde e bem-estar tem alicerce no envelhecimento da população, na alta prevalência de doenças crônicas não transmissíveis (DCNT), no maior acesso à informação, no aumento da renda per capita e na urbanização. ${ }^{2}$ Adicionalmente, $21 \%$ do mercado consumidor de alimentos brasileiro escolhe seus produtos alimentícios considerando esta tendência. ${ }^{2,9}$ Segundo Brasil Food Trends 2020, os consumidores têm preferência pelos produtos que apresentem algum dos seguintes atributos: ${ }^{2}$ redução ou isenção de gordura, açúcar ou sódio; não utilização de corantes ou conservantes adicionados intencionalmente; ser rico em fibras, integral, orgânico, fortificado ou enriquecido com vitaminas e minerais, destinados a restrições alimentares e que não apresentem gordura trans.

Uma alimentação saudável deve ser baseada no consumo de alimentos in natura e minimamente processados. ${ }^{10}$ Mesmo assim, os consumidores buscam os alimentos ultraprocessados, ${ }^{11}$ os quais são claramente contraindicados, visto que seu consumo regular está associado ao aumento da incidência de doenças crônicas não transmissíveis (DCNTs) no Brasil..$^{10,12-14}$ Conforme a definição 
do Guia Alimentar de 2014, os alimentos ultraprocessados são formulações industriais elaboradas com elementos exclusivos desse grupo, como os aditivos alimentares, e apresentam cinco ou mais ingredientes que são substâncias extraídas de alimentos. ${ }^{10}$ Apresentam, ainda, elementos exclusivos dos ultraprocessados, como os aditivos alimentares. ${ }^{10,15}$

Existe uma subcategoria desses alimentos chamada ready to heat ("pronto para aquecer"), cujo consumo substitui as refeições principais (almoço e/ou jantar), como pizzas e lasanhas congeladas. ${ }^{16}$ Esses alimentos podem ser desbalanceados nutricionalmente, pois podem ser ricos em gorduras, açúcar refinado e sódio, ser fabricados com gordura trans, ter baixo teor de fibras e alta densidade energética (de 2,5 a $5 \mathrm{kcal} / \mathrm{g}$ de alimento)..$^{10,17}$

Para uma alimentação saudável, o consumo excessivo de alimentos ultraprocessados é contraindicado..$^{10}$ Apesar disso, estudos apontam o consistente aumento no consumo e aquisição de refeições prontas e misturas industrializas pela população brasileira. . $^{11,15,17-22}$

A tendência de saúde e bem-estar está presente em todos os estratos socioeconômicos. Contudo, destaca-se a classe C, cuja representatividade no Sul do país é de 52,8\% dos consumidores, e em Porto Alegre-RS, 54\% ${ }^{23}$ Conforme o Critério de Classificação Econômica do Brasil (CCEB), a renda domiciliar média desta categoria teve como limite inferior e superior, respectivamente: $\mathrm{R} \$$ $1.625,00$ e R \$2.705,00 por família. ${ }^{23}$ De acordo com a análise socioeconômica da cidade de Porto Alegre, esse estrato econômico reside, predominantemente, em dez regiões/bairros dessa cidade. ${ }^{24}$

A Associação Brasileira das Indústrias da Alimentação (ABIA) revela que pratos congelados com apelação saudável ganham espaço no mercado devido às tendências de conveniência e saúde e bem-estar. ${ }^{6}{ }^{25}$ Segundo a pesquisa Top Of Mind 2016, existem cinco marcas mais lembradas de alimentos ultraprocessados e congelados pela classe econômica C. ${ }^{26}$

O supermercado é o local para aquisição de alimentos das famílias brasileiras, e onde a oferta de produtos alimentícios ultraprocessados é maior. ${ }^{8}$ Por outro lado, devido à crise no consumo, a classe econômica $\mathrm{C}$ tem optado por fazer compras em atacarejos, que são lojas que misturam atacado com apelação de menores preços. ${ }^{27,28}$ Segundo o estudo Top Of Mind 2017, em pesquisa realizada sobre Porto Alegre, existem quatro supermercados e um atacarejo mais lembrados por esse estrato econômico. ${ }^{29}$

Diante do contexto relatado, este estudo pretendeu verificar se produtos alimentícios classificados como ultraprocessados congelados e ready to heat, comercializados na cidade de Porto Alegre-RS, estão sendo fabricados com atributos da tendência de saúde e bem-estar. Para atingir esse objetivo, realizou-se pesquisa observacional descritiva que avaliou parâmetros quantitativos e qualitativos. Adicionalmente, esses parâmetros também foram comparados entre o grupo de marcas mais lembradas (A) e o grupo de outras marcas (B). 


\section{Metodologia}

A coleta de dados desta pesquisa aconteceu de junho a setembro de 2017 na cidade de Porto Alegre-RS, em cinco estabelecimentos comerciais. Trata-se de estudo observacional descritivo de análise de rótulos de produto alimentícios cujos critérios de inclusão foram: ser ultraprocessado, comercializado na forma congelada e ready to heat. Foram comparadas 17 variáveis na amostra geral e 16 parâmetros entre dois grupos: grupo A (composto por cinco marcas mais lembradas de ultraprocessados congelados pela classe econômica C, segundo Top Of Mind 2016) ${ }^{26}$ e grupo B (outras marcas presentes na amostra geral).

\section{Locais de coleta de dados}

Foram escolhidos os quatro supermercados e um atacarejo citados como "mais lembrados pela classe C em Porto Alegre", segundo o Top Of Mind 2017. ${ }^{29}$ As visitas aos locais aconteceram na mesma semana e mês. Visitou-se um estabelecimento de cada grupo comercial em Porto Alegre. A escolha da unidade visitada se baseou na localização predominante da classe econômica C na cidade de Porto Alegre registrada na Análise Socioeconômica Porto Alegre 2017. ${ }^{24}$ A cada gestor dos estabelecimentos, solicitou-se permissão verbal para a coleta de dados.

\section{Coleta de dados}

Foi realizado o registro fotográfico dos seguintes itens presentes no rótulo dos produtos alimentícios: preço unitário praticado no dia, lote, marca, denominação do produto, informação nutricional, peso líquido e lista de ingredientes. A falta de um ou mais desses aspectos configurou critério de exclusão, assim como a ausência de parâmetros da rotulagem nutricional obrigatória. ${ }^{30}$ Também foram dispensados todos os produtos direcionados ao público infantil. Produtos repetidos nos diferentes estabelecimentos foram contabilizados uma única vez. Lotes diferentes de produtos iguais não foram registrados.

\section{Variáveis quantitativas}

As seguintes variáveis quantitativas foram analisadas em cada alimento: preço unitário (\$), peso em gramas da porção, densidade calórica $(\mathrm{kcal} / \mathrm{g})$, relação em porcentagem da participação de gorduras totais no valor energético total (doravante VET), quantidade em gramas de gorduras totais e trans na porção, quantidade em gramas de fibras na porção e em 100g, relação entre 
miligramas de sódio total pelo valor energético total e o número de ingredientes. Essas variáveis foram analisadas na amostra geral e comparadas entre os grupos A e B (exceto preço unitário).

\section{Variáveis qualitativas}

Oito variáveis categóricas, de resposta sim/não, foram analisadas em cada alimento, a saber:

1) excesso de gordura (quando houve $30 \%$ ou mais do VET composto por gorduras totais); ${ }^{31}$

2) excesso de sódio (quando a relação sódio/kcal foi igual ou superior a 1:1);31

3) presença de gordura trans (considerada como gordura trans quando houve indicação numérica superior a zero na tabela nutricional e/ou citação de nomes, na lista de ingredientes, que aponta a presença de gordura trans, $\left.{ }^{(\mathrm{a})}\right) ;^{30,32}$

4) uso de quaisquer corantes adicionados intencionalmente, conforme legislação da Agência Nacional de Vigilância Sanitária (ANVISA); ${ }^{33}$

5) uso de conservantes adicionados intencionalmente segundo ANVISA; ${ }^{33}$

6) redução de ingredientes segundo critério da Resolução da Diretoria Colegiada (RDC) 54 da ANVISA e ingrediente em redução atestado; ${ }^{34}$

7) produto fonte de fibras (critério conforme RDC 54 da ANVISA) 34 ;

8) presença de publicidade no rótulo (dizeres que diferenciam o produto no mercado, como por exemplo: vegetariano, sem lactose, rico em fibras).

Todas essas oito variáveis foram analisadas na amostra geral e comparadas entre os grupos A e B.

(a) Denominações indicativas de gordura trans: ${ }^{32}$ gordura de soja parcialmente hidrogenada; gordura hidrogenada; gordura hidrogenada de soja; gordura parcialmente hidrogenada; gordura parcialmente hidrogenada e/ou interesterificada; gordura vegetal hidrogenada; gordura vegetal parcialmente hidrogenada; margarina; margarina vegetal hidrogenada; óleo de milho hidrogenado;

óleo vegetal de algodão, soja e palma hidrogenado; óleo vegetal hidrogenado; óleo vegetal líquido e hidrogenado; óleo vegetal parcialmente hidrogenado. 


\section{Resultados e Discussão}

A análise estatística foi realizada no software estatístico SPSS versão 18.0. Primeiramente, o teste de Kolmogorov-Smirnov foi aplicado na variável "preço unitário da amostra geral”, com o objetivo de verificar o padrão gaussiano. Devido à anormalidade apontada pelo resultado deste teste, as variáveis quantitativas foram descritas como mediana e intervalo interquartil, pois são medidas não paramétricas pouco afetadas pelos valores individuais. Para comparar as variáveis quantitativas entre os grupos A e B, foi utilizado o teste de Mann-Whitney.

As variáveis qualitativas binomiais foram descritas como frequência absoluta e relativa n (\%) analisadas pelo teste $\mathrm{Z}$ para proporções na amostra geral. Ao comparar essas variáveis categóricas entre os grupos A e B, foi utilizado o teste Qui-Quadrado com correção de Yates, pois este teste estatístico compara as frequências e proporções observadas, e a correção se faz necessária devido ao grau de liberdade 1. Para todos os testes, adotou-se o nível de significância de $\mathrm{p}<0,05$.

Ao todo, 121 produtos alimentícios foram coletados. Neles, aplicou-se o teste de KolmogorovSmirnov na variável "preço unitário", cujo resultado evidenciou o padrão não gaussiano. Assim, essa variável foi descrita como mediana e intervalo interquartil e, com isso, verificou-se que 21 alimentos tinham preços acima do percentil 75, o que distorceria os resultados. Por essa razão, esses 21 produtos foram excluídos da amostra.

O teste de Kolmogorov-Smirnov foi aplicado em 100 produtos alimentícios, e o resultado permaneceu não normal. No entanto, a amostra se tornou mais homogênea, com média $\mathrm{R} \$ 9,77$, mediana de $\mathrm{R} \$ 9,79$, limite superior de $\mathrm{R} \$ 14,90$ e limite inferior de $\mathrm{R} \$ 5,99$. Dessa exclusão, três produtos foram retirados do grupo A (totalizando 56 unidades) e 18 do grupo B (totalizando 44 unidades).

Devido à assimetria na variável "preço", verificada pelo teste de Kolmogorov-Smirnov, os parâmetros quantitativos estão descritos na tabela 1 como mediana e intervalo interquartil.

É possível observar a alta relação de sódio $(\mathrm{mg})$ por kcal, pois $25 \%$ da amostra apresenta 1,95 mg de sódio, ou menos, para cada kcal de alimento. Também se evidencia a alta participação das gorduras totais na composição do valor energético total dos alimentos, com valores iguais ou inferiores a $30,4 \%$ em apenas $25 \%$ da amostra.

Em relação às variáveis qualitativas, por serem categóricas, estas foram analisadas pelo teste $\mathrm{Z}$ para proporções. Segundo o resultado ilustrado na tabela 2, apenas a variável "presença de gordura trans" não foi estatisticamente significativa $(p=0,193)$. 
Tabela 1. Variáveis quantitativas analisadas em amostra de 100 produtos alimentícios classificados como ultraprocessados congelados e ready to heat, comercializados na cidade de Porto Alegre-RS, 2017.

\begin{tabular}{l|c}
\hline \multicolumn{1}{c|}{ Variável quantitativa } & Mediana (p25-p75)* \\
\hline Peso da porção $(\mathrm{g})$ & $130(100-300)$ \\
\hline Densidade energética $(\mathrm{kcal} / \mathrm{g})$ & $1,5(1,2-2,2)$ \\
\hline$\%$ do VET proveniente de gorduras totais & $35,7(30,4-41,7)$ \\
\hline Gorduras totais na porção $(\mathrm{g})$ & $10,5(5,8-17,0)$ \\
\hline Gorduras trans na porção $(\mathrm{g})$ & $0(0-0,5)$ \\
\hline Fibras na porção $(\mathrm{g})$ & $1,8(1,2-2,6)$ \\
\hline Relação sódio kcal & $2,5(1,95-3,1)$ \\
\hline Número de ingredientes & $18(13-23)$ \\
\hline
\end{tabular}

*variáveis quantitativas, descritas como mediana, a qual representa a medida de tendência central e intervalo interquartil em que P75 significa que $75 \%$ da amostra possui valor igual ou inferior ao identificado, e P25 $25 \%$ da amostra, valor igual ou inferior ao indicado.

Tabela 2. Variáveis qualitativas analisadas em amostra de 100 produtos alimentícios classificados como ultraprocessados congelados e ready to heat, comercializados na cidade de Porto Alegre-RS, 2017.

\begin{tabular}{l|c}
\hline \multicolumn{1}{c|}{ Variável categórica } & $\%(\mathrm{n})$ \\
\hline Excesso de gordura* & \\
\hline Sim & $77(77)$ \\
\hline Não & $23(23)$ \\
\hline Excesso de sódio* & \\
\hline Sim & $95(95)$ \\
\hline Não & $5(5)$ \\
\hline Presença de gordura trans & $43(43)$ \\
\hline Presente & $57(57)$ \\
\hline Ausente & continua
\end{tabular}




\begin{tabular}{|c|c|}
\hline Variável categórica & $\%(\mathrm{n})$ \\
\hline \multicolumn{2}{|l|}{ Alimento sem corante* } \\
\hline Sim & $70(70)$ \\
\hline Não & $30(30)$ \\
\hline \multicolumn{2}{|l|}{ Alimento sem conservante* } \\
\hline $\operatorname{Sim}$ & $78(78)$ \\
\hline Não & $22(22)$ \\
\hline \multicolumn{2}{|l|}{ Redução de ingrediente* } \\
\hline $\operatorname{Sim}$ & $11(11)$ \\
\hline Não & $89(89)$ \\
\hline \multicolumn{2}{|l|}{ Light em sódio* } \\
\hline Sim & $11(11)$ \\
\hline Não & $89(89)$ \\
\hline \multicolumn{2}{|l|}{ Fonte de fibras* } \\
\hline $\operatorname{Sim}$ & $26(26)$ \\
\hline Não & $74(74)$ \\
\hline \multicolumn{2}{|l|}{ Publicidade no rótulo* } \\
\hline $\operatorname{Sim}$ & $26(26)$ \\
\hline Não & $74(74)$ \\
\hline
\end{tabular}

*variáveis categóricas binomiais estatisticamente significativas $(\mathrm{p}<0,01)$ descritas como frequência absoluta e relativa $\mathrm{n}(\%)$, analisadas pelo teste $\mathrm{Z}$ para proporções, no qual foi adotado o nível de significância de $\mathrm{p}<0,05$.

É possível observar que as amostras apresentam excesso de sódio (95\%), excesso de gordura (77\%), preferência por não adicionar intencionalmente corantes e conservantes na formulação, conter alimentos considerados light em sódio (11\%) e 74\% não serem fonte de fibras. Além disso, observouse que $26 \%$ das amostras apresentaram publicidade com objetivo de diferenciar seu produto.

Nos rótulos analisados, foram encontrados os seguintes dizeres: "light", "30\% menos sódio", "zero lactose", "integral fonte de fibras", "sem conservantes", "não contém glúten", "fonte de fibras", "baixo índice glicêmico", "não contém gordura trans", "sem adição de conservantes", "com temperos naturais", "vegetariano", "vegano" e "fonte de proteína". 
Quando as variáveis quantitativas foram comparadas entre o grupo A e B, o teste de MannWhitney foi aplicado. Os respectivos resultados são apresentados na tabela 3 .

Tabela 3. Variáveis quantitativas comparadas entre o grupo A e B em amostra de 100 produtos alimentícios classificados como ultraprocessados congelados e ready to heat, comercializados na cidade de Porto Alegre-RS, 2017.

\begin{tabular}{lccc}
\hline \multicolumn{1}{c}{ Variável } & $\begin{array}{c}\text { Grupo A } \\
\text { (mediana (p25-p75) }\end{array}$ & $\begin{array}{c}\text { Grupo B } \\
\text { mediana (p25-p75) }\end{array}$ & P valor \\
\hline Peso da porção $(\mathrm{g})^{*}$ & $175(100-300)$ & $112,50(77-237,5)$ & 0,017 \\
\hline Densidade energética $(\mathrm{kcal} / \mathrm{g})$ & $1,5(1,2-2,3)$ & $1,5(1,25-2,15)$ & 0,920 \\
\hline $\begin{array}{l}\text { \% do VET proveniente de } \\
\text { gorduras totais* }\end{array}$ & $38,7(32,6-42,35)$ & $33,5(28,9-40,2)$ & 0,024 \\
\hline Gorduras totais na porção* $(\mathrm{g})$ & $13(7,85-17,0)$ & $6,65(4,95-15,0)$ & 0,007 \\
\hline Gorduras trans na porção* $(\mathrm{g})$ & $0(0-0,2)$ & $0(1-1,2)$ & 0,036 \\
\hline Fibras na porção* $(\mathrm{g})$ & $2,2(1,5-3,6)$ & $1,3(0,97-1,, 85)$ & $<0,001$ \\
\hline Relação sódio kcal* & $2,65(2,15-3,3)$ & $2,25(1,65-2,8)$ & 0,006 \\
\hline Número de ingredientes* & $20(17-24)$ & $14(10,5-19,5)$ & 0,001 \\
\hline
\end{tabular}

*variáveis estatisticamente significativas analisadas pelo teste de Mann Whitney; adotou-se nível de significância de 0,05 .

É possível observar os parâmetros em que o grupo A obteve valores superiores, comparado ao grupo B, a saber: peso em gramas da porção, \% do VET proveniente de gorduras totais, quantidade em gramas de gorduras totais na porção, quantidade em gramas de fibras na porção relação sódio/ kcal e número de ingredientes. Já o grupo B apresentou quantidades maiores, em gramas, de gordura trans na porção. Não houve diferença estatisticamente relevante entre os grupos sobre a densidade calórica. Vale salientar que o grupo A é composto por quatro das cinco marcas mais lembradas de alimentos ultraprocessados congelados pela classe $\mathrm{C}$, pois uma das marcas não foi encontrada no presente estudo.

Para comparar as variáveis qualitativas entre os grupos A e B, utilizou-se o teste qui quadrado com correção de Yates, conforme apresentado na tabela 4. 
Tabela 4. Variáveis qualitativas comparadas entre o grupo A e B em amostra com 100 produtos alimentícios classificados como ultraprocessados congelados e ready to heat, comercializados na cidade de Porto Alegre-RS, 2017.

\begin{tabular}{|c|c|c|c|}
\hline Variável categórica & $\begin{array}{c}\text { Grupo A } \\
\mathrm{n}(\%)\end{array}$ & $\begin{array}{c}\text { Grupo B } \\
\mathrm{n}(\%)\end{array}$ & $P$ valor \\
\hline Excesso de gordura & & & 0,106 \\
\hline Sim & $47(83,9)$ & $30(68,2)$ & \\
\hline Não & $9(16,1)$ & $14(31,8)$ & \\
\hline Excesso de sódio & & & 0,230 \\
\hline Sim & $55(98,2)$ & $40(90,9)$ & \\
\hline Não & $1(1,8)$ & $4(9,1)$ & \\
\hline Presença de gordura trans & & & 0,520 \\
\hline Presente & $22(39,3)$ & $21(47,7)$ & \\
\hline Ausente & $34(60,7)$ & $23(52,3)$ & \\
\hline Alimento sem corante & & & 0,455 \\
\hline Sim & $37(66,1)$ & $33(75,0)$ & \\
\hline Não & $19(33,9)$ & $11(25,0)$ & \\
\hline Alimento sem conservante & & & 0,566 \\
\hline Sim & $42(75,0)$ & $36(81,8)$ & \\
\hline Não & $14(25,0)$ & $8(18,2)$ & \\
\hline Redução de ingrediente* & & & 0,005 \\
\hline Sim & $11(19,6)$ & $0(0)$ & \\
\hline Não & $45(80,4)$ & $44(100)$ & \\
\hline Light em sódio* & & & 0,005 \\
\hline Sim & $11(19,6)$ & $0(0)$ & \\
\hline Não & $45(80,4)$ & $44(100)$ & \\
\hline Fonte de fibras* & & & 0,006 \\
\hline Sim & $21(37,5)$ & $5(11,4)$ & \\
\hline Não & $35(62,5)$ & $39(88,6)$ & \\
\hline Publicidade no rótulo* & & & 0,023 \\
\hline Sim & $20(35,7)$ & $6(13,6)$ & \\
\hline Não & $36(64,3)$ & $38(86,4)$ & \\
\hline
\end{tabular}

*variáveis categóricas binomiais estatisticamente significativas analisadas pelo teste Qui-Quadrado com correção de Yate; adotou-se nível de significância de 0,05. 
A tabela 4 apresenta as quatro variáveis cujos resultados apontam diferenças significativas entre os grupos. O grupo A apresenta maior número de produtos fontes de fibra, se comparado ao B. No entanto, os dois não podem ser considerados fontes de fibras, pois 89,6\% da amostra do grupo B e 62,5\% do A são negativos para esse parâmetro.

Da mesma forma, é o grupo A que apresenta os produtos alimentícios classificados como light em sódio (apenas 11\% da sua amostra). E, no entanto, apresenta 98,2\% de seus produtos com excesso de sódio. Além disso, embora em ambos os grupos haja poucos produtos com publicidade no rótulo, o grupo A apresenta maior número de produtos alimentícios com dizeres que o diferenciam dos demais, tais como: "30\% menos sódios", "integral, fonte de fibras", "sem conservantes", "vegetariano" e "com temperos naturais".

O presente estudo visou verificar se os produtos alimentícios ultraprocessados congelados e ready to heat comercializados em Porto Alegre-RS estão sendo fabricados com atributos da tendência de saúde e bem-estar. Para que um alimento se enquadre nesta tendência, ele deve apresentar algum dos oito aspectos relevantes elencados por Brasil Food Trends 2020. ${ }^{2}$ No entanto, não se discutiu o item "alimento integral", devido à falta de consenso quanto a essa definição. Também não se avaliou o item "alimento orgânico", pois o conceito de alimento ultraprocessado inviabiliza que este seja considerado orgânico.

Da mesma forma, não houve avaliação quanto a "alimentos fortificados e enriquecidos", pois segundo a Portaria no 31/2002 da ANVISA, a diferenciação entre micronutrientes adicionados com fins nutricionais e com fins de coloração e conservação é muito tênue. Além disso, não se analisou a quantidade de açúcares presentes nos alimentos, porque a definição ready to heat referese a preparações salgadas que substituem as refeições principais almoço e/ou jantar.

Instintivamente, o consumidor compara preço (\$) e peso da porção $(\mathrm{g})$, de modo a calcular um falso "custo benefício". Os resultados desta pesquisa apontam que o peso da porção é superior no grupo de marcas mais lembradas (A). Tendo em vista o maior nível de esclarecimento sobre o comportamento do consumidor desse grupo, tal resultado não surpreende. Porções maiores e de maior densidade calórica estimulam o consumo exacerbado, contribuindo com a epidemia de obesidade e incidência de DCNT.

Segundo o Guia Alimentar para a População Brasileira de 2014, os alimentos ultraprocessados possuem alta densidade energética, variando de 2,5 a $5,0 \mathrm{kcal} / \mathrm{g} .{ }^{10}$ Este estudo, no entanto, apontou valores inferiores. A amostra geral apresentou mediana de 1,5 kcal/g, e não houve diferença estatística entre os grupos. Isso mostra uma mudança na composição desses produtos alimentícios. No entanto, permanecem tendo valor superior ao prato base da alimentação brasileira: arroz e feijão $(1,0 \mathrm{kcal} / \mathrm{g}) \cdot{ }^{10}$ 
Desde 2007, o Ministério da Saúde negocia com a ABIA a redução de gorduras em alimentos processados. ${ }^{35}$ Entretanto, o grupo de alimentos ultraprocessados não foi contemplado nessa reformulação. Observa-se, pelos resultados da atual pesquisa, que $77 \%$ da amostra geral apresenta excesso de gordura - que, segundo critério da Organização Pan-americana da Saúde (OPAS), é quando a participação das gorduras totais na composição do VET é igual ou superior a 30\%. ${ }^{31}$ Além disso, o grupo A apresentou maior quantidade em gramas de gorduras totais na porção, maior porcentagem do VET proveniente de gorduras totais e mais produtos com excesso de gordura (83,9\%, enquanto que o grupo B teve $68,2 \%)$.

Além da quantidade de gordura, é necessário atentar para a quantidade de gordura hidrogenada, uma vez que há forte associação entre consumo de gordura trans e doenças cardiovasculares, pois ela contribui para o desenvolvimento da aterogênese, dislipidemia e desfechos duros. ${ }^{13-14}$ A amostra geral da atual pesquisa evidencia uma quantidade pequena dessa gordura nas porções - $75 \%$ da amostra têm quantidades iguais ou inferiores a $0,5 \mathrm{~g} /$ porção e $50 \%$, inferior a zero. O consumo diário recomendado pela OPAS é de, no máximo, $1 \%$ do VET diário individual. ${ }^{31}$ Em uma dieta de $2.000 \mathrm{kcal}$, a porção diária máxima seria de 2,2g de gordura trans.

Cabe ressaltar que a RDC 360 da ANVISA permite que $0,2 \mathrm{~g}$ de gordura trans seja considerado zero, mas isso não significa que o produto não contenha esse ingrediente. ${ }^{30}$ Assim, avaliou-se quantitativa e qualitativamente a presença da gordura trans, e se percebeu que $43 \%$ dos produtos alimentícios da amostra geral apresentam ingrediente com gordura trans na composição. Resultados semelhantes foram encontrados em outro estudo. ${ }^{32}$ Os resultados da atual pesquisa nos mostram, também, que o grupo B apresentou maior quantidade em gramas de gordura trans na porção.

A ingestão diária de quantidades adequadas de fibras é fundamental, devido a seus efeitos protetores contra doenças cardiovasculares, hipertensão, diabetes, obesidade e câncer de cólon. ${ }^{13,36}$ Preferencialmente, a ingestão de fibras deve ser oriunda de alimentos in natura ou minimamente processados. ${ }^{10}$ No entanto, os consumidores buscam produtos ultraprocessados e seu consumo vem gradualmente aumentando, conforme indicam diversas pesquisas. .11,15, 17-22 $^{2}$

O que os resultados do presente estudo nos mostram é que, embora o grupo A apresente maior quantidade, em gramas, de fibras na porção e número de produtos fontes de fibras (37,5\% ), comparado ao grupo B (11,4\% fonte de fibras), $74 \%$ da amostra geral não se encaixa no critério da RDC 54 da ANVISA para fonte de fibras. ${ }^{34}$ Não sendo, portanto, alimentos de referência para esse parâmetro, concordamos com resultados encontrados por Bieleman ${ }^{17}$ e Laura et al. ${ }^{37}$

No Brasil, em 2014, o Ministério da Saúde firmou o terceiro termo de compromisso com a ABIA para redução de sódio para até 2020 em produtos processados. ${ }^{38}$ No entanto, os ultraprocessados não entraram na lista prioritária do governo para redução. ${ }^{22}$ Os resultados desta pesquisa apontam que $95 \%$ da amostra geral apresenta excesso de sódio, tendo o grupo A maior relação sódio/kcal, 
pois apenas 25\% de sua amostra obteve valor igual ou inferior a 1,95 mg de sódio para cada kcal de alimento, enquanto que o recomendado pela OPAS é de, no máximo, até $1 \mathrm{mg}$ por kcal. Por isso, embora o grupo A tenha produtos com versões light em sódio (19,6\% da sua amostra), 98,2\% de seus produtos permanecem com excesso desse micronutriente. Vale salientar que o alimento light não é sinônimo de saudável, visto que a redução de um elemento pode ser substituída por outro ingrediente de modo excessivo e desbalanceado. ${ }^{39}$

Os resultados obtidos a respeito de sódio, gorduras totais e trans são preocupantes, tendo em vista a contribuição direta desses fatores na incidência e prevalência de DCNT. ${ }^{10,12-14,17}$ A classe $\mathrm{C}$ representa, hoje, aproximadamente $50 \%$ do mercado consumidor, e o consumo de ultraprocessados vem aumentando, segundo dados estatísticos. ${ }^{11,15,17-22} \mathrm{Os}$ consumidores do estrato econômico $\mathrm{C}$ pedem por mudanças nas formulações. ${ }^{40} \mathrm{O}$ consumo regular de produtos com excesso de sódio, gordura, baixo aporte de fibras e que apresentam gordura trans impacta negativamente na saúde de uma classe econômica em ascensão que demanda por produtos mais saudáveis sem abrir mão da conveniência, praticidade e sabor. ${ }^{10,12,40}$

O Guia Alimentar de 2014 traz que os alimentos ultraprocessados possuem mais de cinco ingredientes..$^{10}$ Os resultados da presente pesquisa concordam com essa referência e mostram que o grupo B apresenta menos ingredientes listados, quando comparado ao grupo A. É uma tendência reduzir o número de ingredientes chamada "clean labels". ${ }^{1}$ No entanto, a menor quantidade neste grupo (B) pode ter associação a questões sensoriais e de sabor do produto alimentício.

Assim, ambos os grupos apresentam excesso de gordura e de sódio, por isso a quantidade de ingredientes a mais no grupo A não necessariamente seria de matéria-prima a base de gordura e sal, o que tornaria o produto mais palatável. Vale salientar que este trabalho não avaliou questões sensoriais dos produtos, embora elas de fato existam entre as marcas. É sabido que o grupo A, por ser composto por grandes companhias alimentícias, possui mais recursos financeiros para investir em tecnologia e pesquisa sensorial. Por isso, para se inserir nesse mercado competitivo, é necessário cativar o consumidor pelo sabor.

Os resultados deste estudo mostram a preferência por não adicionar corantes $(70 \%) \mathrm{e}$ conservantes (78\%) intencionalmente na amostra geral, sem diferença estatística entre os grupos. Entretanto, isso não significa que esses alimentos ultraprocessados não tenham esses elementos, devido ao princípio de transferência de aditivos. ${ }^{41}$ As matérias-primas usadas na formulação do produto podem já possuir, em sua composição, corantes e conservantes adicionados, o que torna dispensável relatá-los na lista de ingredientes ou de adicionar no produto final.

Vale ressaltar a importância dos conservantes para assegurar que o alimento seja seguro para o consumo. Na atual conjuntura social, os corantes e conservantes têm sido o foco de diversos estudos, 
que atestam seus possíveis malefícios ao ser humano. ${ }^{42}$ No entanto, antes de um alimento ser "sem conservante" e se adequar às novas tendências, o produto alimentício tem que ser seguro para consumo. ${ }^{41}$ Por isso, o papel dos conservantes vai além de estender a validade e baratear os custos.

\section{Conclusão}

Os resultados deste estudo apontam o panorama geral da amostra, pelo qual é possível identificar atributos da tendência de saúde e bem-estar sendo contemplados pelo grupo de marcas mais lembradas. O grupo A, então, composto por grandes companhias alimentícias, tem mais discernimento sobre tendências de mercado do que o grupo B, das marcas menos lembradas.

Em particular observou-se, para o grupo A, discreta tentativa de se envolver com a tendência de saúde e bem-estar, voltada à classe econômica $\mathrm{C}$, já que apresenta publicidade no rótulo para direcionar seu produto no mercado (35,7\%\% da sua amostra, enquanto que o grupo B, 13,6\%). Este possui alimentos com versões light (19,6\%), tem maior quantidade em gramas de fibras na porção e produtos fontes de fibra, prefere não adicionar intencionalmente corantes e conservantes na fabricação e apresenta menos gordura trans na porção. No entanto, ainda há muito paraprogredir, pois permanecem sendo produtos com excesso de sódio, excesso de gordura, apresentam poucas versões com redução de ingredientese que mantenha o equilíbrio na composição.

\section{Agradecimento}

Agradecemos à Dra. Keila Maria Mendes Ceresér, por suas contribuições metodológicas para o tratamento e análise da base de dados.

\section{Colaboradores}

Kruel JP trabalhou em todas as etapas, desde a concepção do estudo até a revisão da versão final do artigo; foi a campo para coletar os dados da pesquisa para seu trabalho de conclusão de Curso de Nutrição. Gurak PD participou em todas as etapas, desde a concepção do estudo até a revisão da versão final do artigo. Concha-Amin M participou do desenho inicial do estudo e da sua versão final para submissão.

Conflito de interesses: Os autores declaram não haver conflitos de interesse. 


\section{Referências}

1. Kotler P, Keller KL. Coleta de informações e previsão de demanda. In: Kotler P, Keller KL. Administração de marketing. 14 ed. São Paulo: Pearson; 2012. p. 69-95.

2. São Paulo. Governo do Estado. Brasil foodtrends 2020. ITAL; 2010. 171p.

3. Madi L, Amaral R. O que o brasileiro irá comer em 2020?. Revista da ESPM [Internet] 2014; 5. Disponível em: http://www.alimentosprocessados.com.br/arquivos/Consumo-tendencias-einovacoes/O-que-o-brasileiro-ira-comer-em-2020-Artigo-Rev-ESPM.pdf

4. Correia B. Determinantes do consumo de alimentos processados e ultraprocessados em estudantes da Universidade de Brasília (UnB), Distrito Federal. [Trabalho de Conclusão de Curso]. [Brasília]: Universidade de Brasília; 2016.

5. Kruckenfellner J. Indústria ainda pode explorar alimentação saudável no país. DCI - Diário Comércio Indústria \& Serviços [Internet]. 11 mar. 2016. [acesso em: 11 jul. 2017]. Disponível em: http://www. abia.org.br/vns/tmp_2.aspx?id=180\#sthash.h.hMD0VCVQ.bUoCV7Jf.dpbs

6. Coutrin E. Congelados com apelo saudável ganham espaço. DCI - Diário Comércio Indústria \& Serviços [Internet]. 20 out. 2016. Disponível em: https://www.dci.com.br/industria/congeladoscom-apelo-saudavel-ganham-espaco-1.468455

7. Claro RM, Maia EG, Costa BVL, Diniz DP. Preço dos alimentos no Brasil: prefira preparações culinárias a alimentos ultraprocessados. Cad Saúde Pública 2016; 32(8):1-13.

8. Machado PP. Influência dos supermercados na disponibilidade e preço de alimentos ultraprocessados consumidos no Brasil [dissertação]. [São Paulo]: Universidade de São Paulo; 2016.

9. Lima Filho DO, Arca NS, Quevedo-Silva F, Nunes PS, Chung P. Escolha de alimentos: os fatores vida saudável e bem-estar e sensorialidade e prazer. Pensamento e Real. 2016; 31(3):78-87.

10. Saúde M. Guia alimentar para a população brasileira guia alimentar para a população brasileira. 2 ed. Brasília: Secretaria de Atenção à Saúde; 2014.

11. Martins APB, Levy RB, Claro RM, Moubarac JC, Monteiro CA. Participação crescente de produtos ultraprocessados na dieta brasileira (1987-2009). Rev Saúde Pública. 2013; 47(4):656-665.

12. Saúde M. Vigitel Brasil 2016. Brasília: Secretaria de Vigilância em Saúde; 2017.

13. Santos RD, Gagliardi ACM, Xavier HT, Magnoni CD, Cassani R, Lottenberg AMP, et al. I Diretriz sobre consumo de gorduras e saúde cardiovascular. ArqBrasCardiol. 2013; 100(1 Supl. 3):1-40.

14. Souza RJ, Mente A, Maroleanu A, Cozma AI, Ha V, Kishibe T, et al. Intake of saturated and trans unsaturated fatty acids and risk of all cause mortality, cardiovascular disease, and type 2 diabetes: systematic review and meta-analysis of observational studies. Br Med J. 2015; 351(3978):1-16.

15. Monteiro CA, Cannon G, Levy R, Moubarac J-C, Jaime P, Martins AP, et al. O sistema alimentar classificação dos alimentos. World Nutr. 2016; 7(1-3):28-40.

16. Monteiro CA, Levy RB, Claro RM, Castro IRR, Cannon G. Uma nova classificação de alimentos baseada na extensão e propósito do seu processamento. Cad Saúde Pública. 2010; 26(11):2039-2049. 
17. Laura M, Silva D, Li C, Bertazzi R, Li L, Moreira R, et al. Alimentos ultraprocessados e perfil nutricional da dieta no Brasil. Rev Saúde Pública. 2015; 49(38):1-11.

18. Instituto Brasileiro de Geografia e Estatística. Pesquisa de orçamentos familiares 2008-2009: aquisição alimentar domiciliar per capita. Brasília: IBGE; 2009.

19. Instituto Brasileiro de Geografia e Estatística. Pesquisa de orçamentos familiares 2002-2003: aquisição alimentar domiciliar per capita, Brasil e Grandes Regiões. Rio de Janeiro: IBGE; 2004.

20. Instituto Brasileiro de Geografia e Estatística. Pesquisa de orçamentos familiares 2002-2003: perfil das despesas no Brasil. Brasília: IBGE; 2004.

21. Instituto Brasileiro de Geografia e Estatística. Pesquisa de orçamentos Familiares 2008-2009: despesas, rendimentos e condições de vida. Rio de Janeiro: IBGE; 2009.

22. Martins APB. Redução de sódio em alimentos: uma análise dos acordos voluntários no Brasil. São Paulo: Instituto Brasileiro de Defesa do Consumidor; 2014.

23. Associação Brasileira de Empresas de Pesquisa. Critério Brasil 2015 e atualização da distribuição de classes para 2016 [Internet]. Disponível em: http://www.abep.org/criterio-brasil

24. Ferreira S, Menezes B. Análise socioeconômica da cidade de Porto Alegre. Porto Alegre: Fundação de Economia e Estatística; 2017.

25. Lucchese-Cheung T. Atitudes do consumidor em relação a um produto alimentar: tendências do setor de alimentos congelados. Universidade FUMEC. 2015; 17(1 ):61-81.

26. Coca-Cola, Omo, Nike, Nestlé e Samsung lideram Top of Mind 2016. Data Folha [Internet]. 26 out. 2016. Disponível em: http://datafolha.folha.uol.com.br/opiniaopublica/2016/10/1826495-coca-colaomo-nike-nestle-e-samsung-lideram-top-of-mind-2016.shtml

27. Chiara M. Atacarejo cresce mais que o dobro do varejo. Estadão [Internet] 29 abr. 2013. Disponível em: http://economia.estadao.com.br/noticias/negocios, atacarejo-cresce-mais- que-o-dobro-dovarejo, $152280 \mathrm{e}$

28. Mattos A. Atacarejo tem mais clientes que supermercados no país. Valor Econômico [Internet] 23 nov. 2016. Disponível em: http://www.valor.com.br/empresas/4784481/atacarejo-tem-mais-clientesque-supermercados-no-pais

29. Top of Mind Amanhã 2017. Especial Amanhã. Porto Alegre; 2017. Disponível em: http:// topofmindamanha.com.br/caderno_top_2017.pdf

30. Brasil. Anvisa. Resolução no 360, de 23 de dezembro de 2003. Aprova Regulamento Técnico sobre Rotulagem Nutricional de Alimentos Embalados, tornando obrigatória a rotulagem nutricional. Diário Oficial da União. 26 dez. 2003.

31. Organização Pan-Americana da Saúde. Modelo de perfil nutricional da Organização Pan-Americana da Saúde. Washington, DC: OPAS; 2016. 38 p.

32. Silveira BM. Informação alimentar e nutricional da gordura trans em rótulos de produtos alimentícios industrializados [dissertação]. [Florianópolis]: Universidade Federal de Santa Catarina, Centro de Ciências da Saúde, Programa de Pós-Graduação em Nutrição; 2011. 
33. Agência Nacional de Vigilância Sanitária. Classificação dos corantes caramelos II, III e IV e dos demais corantes autorizados para uso em alimentos. Informe Técnico no. 68/2015. Brasília: ANVISA; 2015.

34. Brasil. Anvisa. RDC no 54 de 12 de novembro de 2012. Dispõe sobre o Regulamento Técnico sobre Informação Nutricional Complementar. Diário Oficial da União. 13 nov. 2012.

35. Brasil. Ministério da Saúde. Ministério da Saúde e Abia fecham acordo para reduzir teor de sódio em carnes e laticínios [Internet]. 29 jul. 2014 Disponível em: http://www.brasil.gov.br/saude/2013/11/ ministerio-da-saude-e-abia-fecham-acordo-para-reduzir-teor-de-sodio-em-carnes-e-laticinios

36. Sarmento F, Bernaud R, Rodrigues TC. Fibra alimentar: Ingestão adequada e efeitos sobre a saúde do metabolismo. ArqBrasEndocrionolMetab. 2013; 57(6):397-405.

37. Bielemann RM, Minten GC, Horta BL, Gigante DP. Consumo de alimentos ultraprocessados e impacto na dieta de adultos jovens. Rev Saúde Pública. 2015; 49(28):10.

38. Brasil. Ministério da Saúde. Termo de Compromisso que firmam entre si a União, por intermédio do Ministério da Saúde e a Associação Brasileira das Indústrias de Alimentação- ABIA, com a finalidade de estabelecer metas nacionais para a redução do teor de sódio em alimentos processados no Brasil. Diário Oficial da União. 28 ago. 2012.

39. Castro MRP, Lima FD. Comparação de rótulos: ingredientes de produtos Light $\mathrm{x}$ Tradicionais [trabalho de conclusão de curso]. [Brasília]: Centro Universitário de Brasília, Faculdade de Ciências da Educação e Saúde; 2017.

40. Silva A. O comportamento dos consumidores da classe $\mathrm{C}$ em relação à alimentação saudável. Rio de Janeiro: Pontifícia Universidade Católica do Rio de Janeiro; 2016.

41. Brasil. Anvisa. Portaria no 540, de 27 de outubro de 1997. Aprova o Regulamento Técnico: Aditivos Alimentares - definições, classificação e emprego. Diário Oficial da União. 28 out. 1997.

42. Sá P, Ferreira F, Vila Nova R, Mourão T, Andrade V, Andrade Â, et al. Uso abusivo de aditivos alimentares e transtornos de comportamento: há uma relação? Int J Nutrology. 2016; 9(2):7.

Recebido: 26 de janeiro, 2018

Revisado: 05 de fevereiro, 2018

Aceito: 05 de março, 2018 